Please do not remove this page

RMIT

UNIVERSITY

\title{
Correlated imaging visibility using a parametric downconversion source seeded with partially coherent stimulation
}

Castelletto, Stefania; Roberts, Ann; Scholten, R

https://researchrepository.rmit.edu.au/esploro/outputs/9921858618801341/filesAndLinks?institution=61RMIT_INST\&index=null

Castelletto, S., Roberts, A., \& Scholten, R. (2009). Correlated imaging visibility using a parametric downconversion source seeded with partially coherent stimulation. Journal of the Optical Society of America B-Optical Physics, 26(6), 1221-1227. https://doi.org/10.1364/JOSAB.26.001221

Document Version: Published Version

Published Version: https://doi.org/10.1364/JOSAB.26.001221

Repository homepage: https://researchrepository.rmit.edu.au

(C) 2009 Optical Society of America

Downloaded On 2023/04/27 00:42:48 +1000

Please do not remove this page 
Thank you for downloading this document from the RMIT Research Repository.

The RMIT Research Repository is an open access database showcasing the research outputs of RMIT University researchers.

RMIT Research Repository: http://researchbank.rmit.edu.au/

\section{Citation:}

Castelletto, S, Roberts, A and Scholten, R 2009, 'Correlated imaging visibility using a parametric downconversion source seeded with partially coherent stimulation', Journal of the Optical Society of America B-Optical Physics, vol. 26, no. 6, pp. 1221-1227.

See this record in the RMIT Research Repository at:

http://researchbank.rmit.edu.au/view/rmit:21949

Version: Published Version

Copyright Statement: (c) 2009 Optical Society of America

Link to Published Version:

http://dx.doi.org/10.1364/JOSAB.26.001221 


\title{
Correlated imaging visibility using a parametric downconversion source seeded with partially coherent stimulation
}

\author{
S. Castelletto, ${ }^{*}$ A. Roberts, and R. E. Scholten \\ School of Physics, The University of Melbourne, 3010 Victoria, Australia \\ *Corresponding author: sacas@unimelb.edu.au
}

Received January 23, 2009; revised April 13, 2009; accepted April 19, 2009; posted April 21, 2009 (Doc. ID 106539); published May 14, 2009

\begin{abstract}
We have studied a correlated imaging scheme using parametric downconversion stimulated with a partially coherent beam as a source. We analyze the dependence of quantum spatial correlations and image reconstruction features on the crystal length, the coherence length, and the transverse size of the seed beam in low- and high-gain regimes of parametric downconversion. We show that the visibility of ghost interference can be adjusted by varying the intensity and/or the coherence length of the seed, with improved visibility in the highgain regime, and a visibility greater than 0.5 in the low-gain regime. () 2009 Optical Society of America

OCIS codes: $190.4410,270.0270,270.4180$.
\end{abstract}

\section{INTRODUCTION}

Classical coherence-function imaging is a well-known technique dating back to Hanbury-Brown and Twiss interferometry [1]. The coherence function of a source $G^{(1)}\left(\mathbf{x}_{1}, \mathbf{x}_{2}\right)=\left\langle\mathbf{E} *\left(\mathbf{x}_{1}\right) \mathbf{E}\left(\mathbf{x}_{2}\right)\right\rangle$ is determined from measurement of the intensity correlations at two distant detection planes in the propagated field, i.e., from measurement of the fourth-order correlation function, $G^{(2)}\left(\mathbf{x}_{1}, \mathbf{x}_{2}\right)$. The light is assumed thermal, so that the Siegert relation applies:

$$
G^{(2)}\left(\mathbf{x}_{1}, \mathbf{x}_{2}\right)=G^{(1)}\left(\mathbf{x}_{1}, \mathbf{x}_{1}\right) G^{(1)}\left(\mathbf{x}_{2}, \mathbf{x}_{2}\right)+\left|G^{(1)}\left(\mathbf{x}_{1}, \mathbf{x}_{2}\right)\right|^{2} .
$$

In more recent years [2,3], the spatial quantum correlation of entangled photons generated in parametric downconversion (PDC) has been used to demonstrate "ghost imaging" and "ghost interference" by measuring coincident photon pairs. The interesting point of these experiments, with respect to the classical coherence-function imaging technique, is the possibility of performing the measurement in the absence of single-channel noise. Once the two-photon imaging technique was established, it was believed possible only with an entangled photon source, relying on a direct link between the fourth-order correlation function and the biphoton field amplitude, $G^{(2)}\left(\mathbf{x}_{1}, \mathbf{x}_{2}\right)=\left|\psi_{12}\left(\mathbf{x}_{1}, \mathbf{x}_{2}\right)\right|^{2}$.

The association of ghost imaging with entanglement fostered the possibility of improved resolution and visibility compared with classical coherent imaging techniques, with potential applications in metrology, quantum holography, and lithography. However, it became clear [4] that entangled photons generated by PDC exhibit properties that are analogous to those of ordinary photons that are generated by incoherent sources, and a duality between partial coherence and partial entanglement was established [5]. The nature of ghost imaging has triggered many interesting debates about the necessity of the en- tanglement [6-15]. At present, the idea that both classical thermal sources and quantum entangled beams can be used for ghost imaging and ghost diffraction is widely accepted. In order to realize the practical use of correlated imaging, further investigation of its properties and the characteristics of the source is essential. Gatti et al. [10] compared image visibility in the classical and quantum regimes and showed that the visibility in the classical regime can be, at most 0.5 , while in single-photon pair detection with entangled photons it can reach unity. D'Angelo et al. [16] have compared the resolution of quantum and classical ghost imaging, showing that higher resolution is, in principle, possible with an entangled source. Meanwhile, Ferri et al. [17] experimentally demonstrated the highest resolution of ghost imaging and ghost diffraction with a thermal light, and they demonstrated ghost imaging with homodyne detection [18]. Coherent imaging of a pure phase object is possible with both quantum and classical incoherent light [19]. By designing specific incoherent light sources using scattering media, it is possible to use near-field scattering, in which the speckle size is governed solely by the properties of the scattering medium, to enhance the resolution of ghost imaging $[19,20]$. Some questions about ghost imaging still remain open, such as the dependency of the visibility and resolution on the complexity of the object [21], on the particular optical setup used (e.g., lensless ghost imaging for applications such as X-ray imaging), and on the type of source used, particularly with respect to stimulated PDC sources [22].

A novel source for implementing ghost imaging has recently been demonstrated by using an intense field in a seeded multimode PDC system [23]. Despite the thermal injected seed, the source maintains some of the features typical of a PDC ghost-imaging setup; for example, the quantum thin-lens equation is applicable, being governed by the phase-matching function of the nonlinear crystal. However, the specific experimental example considered in 
[23] exhibits the visibility and resolution expected for a thermal source, despite the residual quantum correlation [24]. Relative to the visibility of ghost interference with a multimode optical parametric amplifier, the visibility drops from unity to near zero with an increase in the parametric gain of the amplifier [25] due to the presence of experimental factors such as the photon bandwidth and the coincidence time set by the detection system. In addition, when a large number of modes are involved in the parametric process, the visibility tends to a maximum value of 0.2 [26]. Another difficulty is with the magnitude of two-photon counts, when parametric downconversion alone is used. Stimulated processes can enhance the count rate to allow measurements with nonintensified CCD cameras. The use of stimulated, as well as spontaneous, processes can therefore potentially improve practical quantum imaging in terms of resolution and visibility.

In this paper we analyze in more detail the properties of PDC stimulated by a partially coherent source, in terms of quantum spatial correlation for low parametric gain (two-mode operation), and for a high-gain regime (multiphoton and multimode operation). We show that, in these circumstances, the source exhibits a ghost interference pattern and visibility that can vary from that expected with partially coherent to pure entangled photon sources. The transition can be controlled by varying the intensity and the coherence length of the injected source. The properties of ghost interference are analyzed and compared with the classical and quantum cases.

\section{THEORY}

Figure 1 shows the principle for two-photon correlated imaging. For a completely classical source, a 50:50 beamsplitter (BS) is used in conjunction with a partially coherent or completely incoherent input, with field operator $\mathbf{a}_{1}$. Instead, entangled photon pairs can be generated via $\mathrm{PDC}$, if the BS is replaced with a nonlinear crystal (NLC) driven by a pump laser. We consider the situation where the input field $\mathbf{a}_{1}$ is a partially coherent source.

In either case, the second input of the BS or NLC is taken to be the vacuum state $\mathbf{v}$. The output biphoton field then propagates through two optical paths with impulse response functions $h_{j}\left(x_{j}, x_{j}^{\prime}\right) ; j=1,2$. For simplicity we assume that $x$ is one dimensional. In any correlated imaging experiment, the directly measured quantity is the fourthorder correlation function $G^{(2)}\left(x_{1}^{\prime}, x_{2}^{\prime}\right)$ at the detection plane between a bucket detector, positioned in the arm where the object is placed, and a position-sensitive detector located in the reference arm. For a classical source, the information about the object is contained in the term $\Delta G\left(x_{1}^{\prime}, x_{2}^{\prime}\right)=\left|G^{(1)}\left(x_{1}^{\prime}, x_{2}^{\prime}\right)\right|^{2}$, while for an entangled photon source the information affects $\left|\psi_{12}\left(x_{1}^{\prime}, x_{2}^{\prime}\right)\right|^{2}$. The biphoton amplitude probability at the detection plane is given by $\psi_{12}\left(x_{1}^{\prime}, x_{2}^{\prime}\right)$, where the suffix 12 indicates that PDC field amplitude corresponds to a second-order cross-correlation function. This function $\psi_{12}\left(x_{1}, x_{2}\right)=\left\langle 0,0\left|\hat{E}_{1}^{+}\left(x_{1}\right) \hat{E}_{2}^{+}\left(x_{2}\right)\right| \Psi\right\rangle$, where $|\Psi\rangle$ is the output state of the photons. In the lowgain regime, $|\Psi\rangle$ can be limited to the first order of the interaction Hamiltonian, giving a vacuum state and an entangled two-photon state. In the high-gain regime,
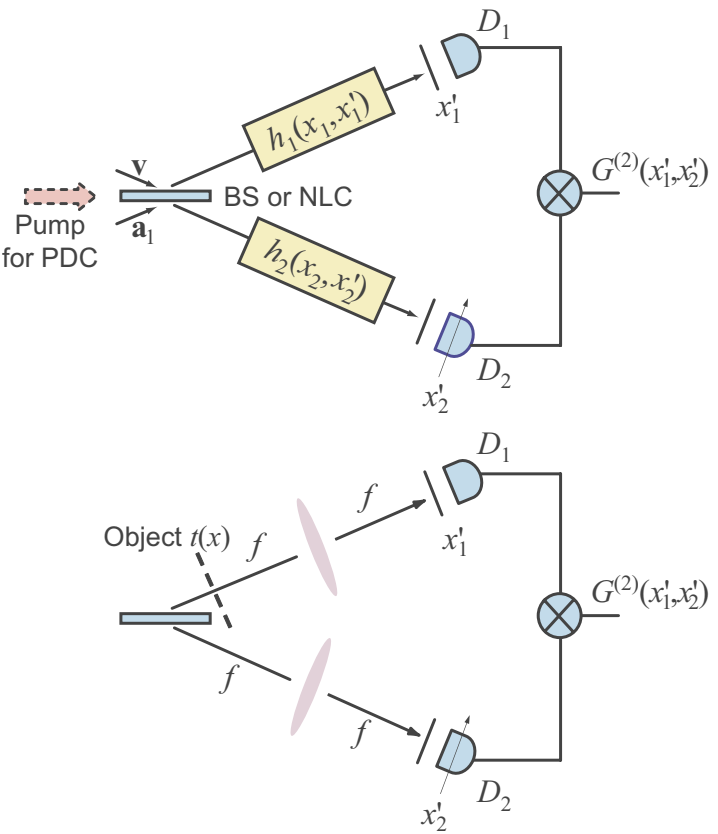

Fig. 1. (Color online) Schematic showing the principle of correlated imaging using either a classical or entangled source. For the classical source, a beamsplitter (BS) is used to generate twin beams from an input beam indicated as $\mathbf{a}_{1}$. For stimulated PDC a nonlinear crystal (NLC) replaces the $\mathrm{BS}$, and the crystal is pumped by a strong input beam plus a seed, again indicated by $\mathbf{a}_{1}$. The vacuum state $\mathbf{v}$ is assumed for the other port of the BS or NLC. The input response functions of the two beams as generated after the BS or the NLC, $h_{j}\left(x_{j}, x_{j}^{\prime}\right) j=1,2$, describe the propagation of the beams to the detection planes. The lower figure shows the imaging arrangement with object of transmission function $t(x)$ and lens of focal length $f$.

multiphoton production has to be considered, and this approximation is no longer valid. The duality between partial coherence and entanglement is summarized by the correspondence between the biphoton probability amplitude and the coherence function $G^{(1)}\left(x_{1}, x_{2}\right)$. A more rigorous approach, where the field operators are considered, relates the $G^{(1)}\left(x_{1}, x_{2}\right)$ of a thermal source, characterized by Gaussian statistics, to the mean photon number per mode of transverse wavevector $q$ [10]:

$$
G^{(1)}\left(x_{1}, x_{2}\right)=\frac{1}{(2 \pi)} \int \mathrm{d} q e^{-i q\left(x_{1}-x_{2}\right)}\langle n(q)\rangle_{\text {therm }} .
$$

For a thermal source characterized by Gaussian statistics, using the field operator approach, the autocorrelation function of any order can be written in terms of the second-order autocorrelation function. The same simplification occurs for a PDC process, and the unitary inputoutput transformations relating the field operators at the output face of the crystal $\mathbf{b}$ to the input operators $\mathbf{a}$ are given by

$$
\mathbf{b}_{i}(q)=U_{i}(q) \mathbf{a}_{i}(q)+V_{i}(q) \mathbf{a}_{j}^{\dagger}(-q), \quad i, j=1,2 ; i \neq j .
$$

The gain functions $U_{i}(q), V_{i}(q)$ for the high-gain regime have been calculated analytically for the case of a planewave stationary pump and numerically using a stochastic model based on an equivalent Wigner representation in phase space [27], to account for the transverse finite dimension of the pump. The gain coefficients are respon- 
sible for both the spectral and spatial quantum correlation; however, in this paper we do not account for the frequency dependence, assuming that the measurement time is shorter than the temporal coherence time of the source. Also in the PDC case the second-order crosscorrelation function in the near field can be written in terms of the gain functions as follows:

$$
\Gamma_{12}^{(1)}\left(x_{1}, x_{2}\right)=\frac{1}{2 \pi} \int \mathrm{d} q e^{i q\left(x_{1}-x_{2}\right)} U_{1}(q) V_{2}(-q),
$$

and the second-order autocorrelation function is given by

$$
\Gamma_{j j}^{(1)}\left(x_{1}, x_{2}\right)=\frac{1}{2 \pi} \int \mathrm{d} q e^{-i q\left(x_{1}-x_{2}\right)}\left|V_{j}(-q)\right|^{2}, \quad j=1,2 .
$$

By following the same reasoning it is easy to prove that, for a seed with thermal statistics, the second-order crosscorrelation function becomes [24]

$$
\begin{aligned}
\Gamma_{12}^{(1)}\left(x_{1}, x_{2}\right)= & \frac{1}{2 \pi} \int \mathrm{d} q e^{i q\left(x_{1}-x_{2}\right)} U_{1}(q) \\
& \times V_{2}(-q)\left(1+\langle n(-q)\rangle_{\text {therm }}\right),
\end{aligned}
$$

and the second-order autocorrelation functions are, assuming the seed is in arm 1 ,

$$
\begin{aligned}
\Gamma_{11}^{(1)}\left(x_{1}, x_{2}\right)= & \frac{1}{2 \pi} \int \mathrm{d} q e^{-i q\left(x_{1}-x_{2}\right)}\left[\left|V_{1}(-q)\right|^{2}\left(1+\langle n(q)\rangle_{\text {therm }}\right)\right. \\
& \left.+\langle n(q)\rangle_{\text {therm }}\right] \\
\Gamma_{22}^{(1)}\left(x_{1}, x_{2}\right)= & \frac{1}{2 \pi} \int \mathrm{d} q e^{-i q\left(x_{1}-x_{2}\right)}\left|V_{2}(-q)\right|^{2}\left(1+\langle n(q)\rangle_{\text {therm }}\right)
\end{aligned}
$$

\section{PARTIALLY COHERENT SEED}

A thermal source can be modelled as a partially coherent source with a finite transverse size with second-order autocorrelation function given by the Gaussian-Schell model,

$$
\begin{aligned}
G^{(1)}\left(x_{1}, x_{2}\right)= & \left\langle n_{\text {seed }}\right\rangle \exp \left[-\left(x_{1}-x_{2}\right)^{2} / 2 l_{\text {seed }}^{2}\right] \\
& \times \exp \left[-\left(x_{1}^{2}+x_{2}^{2}\right) / 4 w_{\text {seed }}^{2}\right],
\end{aligned}
$$

where $w_{\text {seed }}$ is the transverse dimension of the source intensity, $l_{\text {seed }}$ is the transverse coherence length, and $\left\langle n_{\text {seed }}\right\rangle$ is the mean photon number. The second-order correlation function can then also be written in terms of the transverse $k$-vector component $q$, and when Fourier transformed with respect to $\left(x_{1}-x_{2}\right)$ becomes

$$
\widetilde{G}^{(1)}\left(x_{1}, x_{2}, q\right) \propto \exp \left[-\left(x_{1}+x_{2}\right)^{2} / 8 w_{\text {seed }}^{2}\right]\left\langle n_{\text {seed }}(q)\right\rangle,
$$

where $\left\langle n_{\text {seed }}(q)\right\rangle=\left\langle n_{\text {seed }}\right\rangle \exp \left[-q^{2} l_{1}^{2}\right]$ is the mean photon number per mode $q$ and $l_{1}=\sqrt{2} l_{\text {seed }} w_{\text {seed }}\left(l_{\text {seed }}^{2}+4 w_{\text {seed }}^{2}\right)^{-1 / 2}$. We refer now to Fig. 1 for the case of a classical source, where a BS generates two identical beams from a partially coherent source, and in particular the arrangement of [10]. An object of transmittance $t(x)$ is placed in arm 1 close to the source. A lens, focal length $f$, is placed in each arm, midway between source and detection plane, which are separated by distance $2 f$. It is easy to show that the measured correlation function obtained by propagating the autocorrelation function of a partially coherent source to the detection plane is given by

$$
\begin{aligned}
\Delta G_{\text {seed }}\left(x_{1}^{\prime}, x_{2}^{\prime}\right)= & \mid \frac{1}{2 \pi} \int \mathrm{d} x_{1} \mathrm{~d} x_{2} \mathrm{~d} q h_{1}^{*}\left(x_{1}, x_{1}^{\prime}\right) h_{2}\left(x_{2}, x_{2}^{\prime}\right) e^{-i q\left(x_{1}-x_{2}\right)} \\
& \left.\times \widetilde{G}^{(1)}\left(x_{1}, x_{2}, q\right)\right)\left.\right|^{2},
\end{aligned}
$$

and completing the calculation

$$
\begin{aligned}
\Delta G_{\text {seed }}\left(x_{1}^{\prime}, x_{2}^{\prime}\right) & \\
\cong & N_{o}^{2} \mid\left\langle n_{\text {seed }}\left[\frac{-\sqrt{2} \pi x_{2}^{\prime}}{f \lambda}\left(\frac{l_{\text {seed }}^{2}+4 w_{\text {seed }}^{2}}{l_{\text {seed }}^{2}+2 w_{\text {seed }}^{2}}\right)^{1 / 2}\right]\right\rangle \\
& \times\left.\widetilde{T}\left\lfloor\frac{2 \pi}{f \lambda}\left\{\left(\frac{2 w_{\text {seed }}^{2}-l_{1}^{2}}{2 w_{\text {seed }}^{2}+l_{1}^{2}}\right) x_{2}^{\prime}-x_{1}^{\prime}\right\}\right]\right|^{2},
\end{aligned}
$$

with $\widetilde{T}(q)=\int \mathrm{d} q t(x) \exp (-i q x) / \sqrt{2 \pi}$. For the impulse response function of arm 1 we have used $h_{1}\left(x_{1}, x_{1}^{\prime}\right)$ $=-i t\left(x_{1}\right) \exp \left(-2 \pi i x_{1} x_{1}^{\prime} / f \lambda\right) / \sqrt{f \lambda}$, and in arm 2, $h_{2}\left(x_{2}, x_{2}^{\prime}\right)$ $=-i \exp \left(-2 \pi i x_{2} x_{2}^{\prime} / f \lambda\right) / \sqrt{f \lambda}$. $N_{o}$ is a factor depending on $l_{1}$ and $w_{\text {seed }}$. In comparison, for a perfectly incoherent source with infinite transverse dimension, the correlation function is simply [10]

$$
\Delta G\left(x_{1}^{\prime}, x_{2}^{\prime}\right) \propto\left|\left\langle n_{\text {therm }}\left(-\frac{2 \pi x_{2}^{\prime}}{f \lambda}\right)\right\rangle \widetilde{T}\left[\frac{2 \pi}{f \lambda}\left(x_{2}^{\prime}-x_{1}^{\prime}\right)\right]\right|^{2} .
$$

\section{SEEDED PARAMETRIC DOWNCONVERSION SOURCE}

A PDC source can be treated as an incoherent source, and the spatial correlation then can be found from Eq. (6). Here we consider the plane-wave stationary pump approximation, with perfect transverse momentum correlation. The key parameters are the amplitude of the pump field $\alpha_{p}$, the length of the crystal $(L)$, and the parametric gain $\sigma_{p} \propto \alpha_{p} \chi L$, which depends on the effective nonlinear coefficient $\chi$. The low-gain regime corresponds to $\sigma_{p} \ll 1$, while for high gain, $\sigma_{p} \gg 1$. We can calculate the correlation function at the detection plane with impulse response functions as in Section 3, for the simple case of an incoherent source as seed, from Eq. (6):

$$
\begin{aligned}
& \Delta G_{\text {PDC+seed }}\left(x_{1}^{\prime}, x_{2}^{\prime}\right) \\
& =\mid \frac{1}{2 \pi} \int \mathrm{d} x_{1} \mathrm{~d} x_{2} \mathrm{~d} q h_{1}\left(x_{1}, x_{1}^{\prime}\right) h_{2}\left(x_{2}, x_{2}^{\prime}\right) e^{i q\left(x_{1}-x_{2}\right)} \\
& \quad \times U_{1}(q) V_{2}(-q) \times\left.\left[1+\left\langle n_{\text {therm }}(-q)\right\rangle\right]\right|^{2} .
\end{aligned}
$$

Completing the integration over $q$, 


$$
\begin{aligned}
\Delta G_{\text {PDC }+ \text { seed }}\left(x_{1}^{\prime}, x_{2}^{\prime}\right) \\
=\mid \frac{\sqrt{2 \pi}}{(f \lambda)^{2}} U_{1}\left(\frac{2 \pi x_{2}^{\prime}}{f \lambda}\right) V_{2}\left(\frac{-2 \pi x_{2}^{\prime}}{f \lambda}\right) \widetilde{T}\left[\frac{2 \pi}{f \lambda}\left(x_{1}^{\prime}+x_{2}^{\prime}\right)\right] \\
\\
\quad \times\left.\left(1+\left\langle n_{\text {therm }}\left(\frac{-2 \pi x_{2}^{\prime}}{f \lambda}\right)\right\rangle\right)\right|^{2}
\end{aligned}
$$

In Eq. (14) the functions $U, V$ and $\left\langle n_{\text {therm }}\right\rangle$ can be considered constant in $q$ because the transverse coherence of the sources is negligible. However when a pseudothermal source is used, specifically a partially coherent source, the transverse coherence of the seed must be considered. If the seed is treated as a partially coherent source as in Eq. (11), the correlation function at the detection plane, for impulse response functions as in Section 3, can be calculated as

$$
\begin{aligned}
& \Delta G_{\text {PDC+seed }}\left(x_{1}^{\prime}, x_{2}^{\prime}\right) \\
& =\mid \frac{1}{2 \pi} \int \mathrm{d} x_{1} \mathrm{~d} x_{2} \mathrm{~d} q h_{1}\left(x_{1}, x_{1}^{\prime}\right) h_{2}\left(x_{2}, x_{2}^{\prime}\right) e^{i q\left(x_{1}-x_{2}\right)} \\
& \quad \times U_{1}(q) V_{2}(-q) \times\left.\left[1+\widetilde{G}^{(1)}\left(x_{1}, x_{2},-q\right)\right]\right|^{2} .
\end{aligned}
$$

The first term in Eq. (16) is due to quantum correlation while the second term describes the spatial correlation influence of the seed to the final resolution and visibility. By integrating over the spatial variables we obtain

$$
\begin{aligned}
\Delta G_{\mathrm{PDC}+\text { seed }}\left(x_{1}^{\prime}, x_{2}^{\prime}\right)= & \mid \frac{\sqrt{2 \pi}}{(f \lambda)^{2}} U_{1}\left(\frac{2 \pi x_{2}^{\prime}}{f \lambda}\right) V_{2}\left(\frac{-2 \pi x_{2}^{\prime}}{f \lambda}\right) \\
& \times \widetilde{T}\left[\frac{2 \pi}{f \lambda}\left(x_{1}^{\prime}+x_{2}^{\prime}\right)\right]+\left.g\left(x_{1}^{\prime}, x_{2}^{\prime}\right)\right|^{2},
\end{aligned}
$$

where

$$
\begin{aligned}
g\left(x_{1}^{\prime}, x_{2}^{\prime}\right)= & \frac{2 \sqrt{2 \pi}}{(f \lambda)^{2}} w_{\text {seed }} \int \mathrm{d} q U_{1}(q) V_{2}(-q)\left\langle n_{\text {seed }}(-q)\right\rangle \\
& \times \exp \left(-2 w_{\text {seed }}^{2}\left(-2 \pi x_{2}^{\prime}+f \lambda q\right)^{2} / f^{2} \lambda^{2}\right) \\
& \times \widetilde{T}\left[\frac{2 \pi}{f \lambda}\left(x_{1}^{\prime}-x_{2}^{\prime}\right)+2 q\right] .
\end{aligned}
$$

For high $\alpha_{p}$ and short crystals, the function $U_{1}(q) V_{2}(-q)$ becomes broader, corresponding to a shorter transverse coherence of the PDC source. The transverse coherence of the PDC scales with the square root of the crystal length, due to photon diffraction along the crystal, and in most cases is much shorter than the seed transverse coherence and smaller than the object transverse dimensions. Then the function $U_{1}(q) V_{2}(-q)$ can be considered constant and the calculation performed analytically. The calculation was performed both numerically and analytically, where possible, to confirm that results are the same for both methods.

\section{DIFFRACTION PATTERN RECONSTRUCTION}

We studied the diffraction pattern reconstruction of a double slit for different values of $l_{\text {seed }}$ and for different intensities of the seed. In the reconstruction we assumed that the detector in $x_{1}^{\prime}$ is a point detector. Results are shown in Fig. 2 for the case of stimulated PDC in a type I BBO crystal, generating degenerate photons at $702 \mathrm{~nm}$ in an almost collinear configuration, for a fixed transverse dimension of the partially coherent seed. In varying the intensity of the seed, the stimulated PDC exhibits a ghost diffraction pattern that lies between that for pure PDC and partially coherent sources. Thus for high intensity of the injected seed $\left(\left\langle n_{\text {seed }}\right\rangle=1000\right)$ the stimulated PDC behaves as a partially coherent source, while for a low number of photons per mode $\left(\left\langle n_{\text {seed }}\right\rangle=0.01\right)$, the diffraction pattern is equivalent to that for PDC. For intermediate values of $\left\langle n_{\text {seed }}\right\rangle$ the source presents intermediate properties.

Figure 2 also shows the normalized $\Delta G_{\mathrm{PDC}+\text { seed }}\left(x_{1}^{\prime}\right.$ $\left.=0, x_{2}^{\prime}\right)$ for a fixed $\sigma_{p}=0.1$ and for two different coherence lengths of the seed, $l_{\text {seed }}=40$ and $20 \mu \mathrm{m}$. The parameter $\left\langle n_{\text {seed }}\right\rangle$ is varied from 0.01 to 1000 , and the same functions are plotted for both pure PDC and partially coherent sources. By increasing $\left\langle n_{\text {seed }}\right\rangle$, the curves obtained by us-

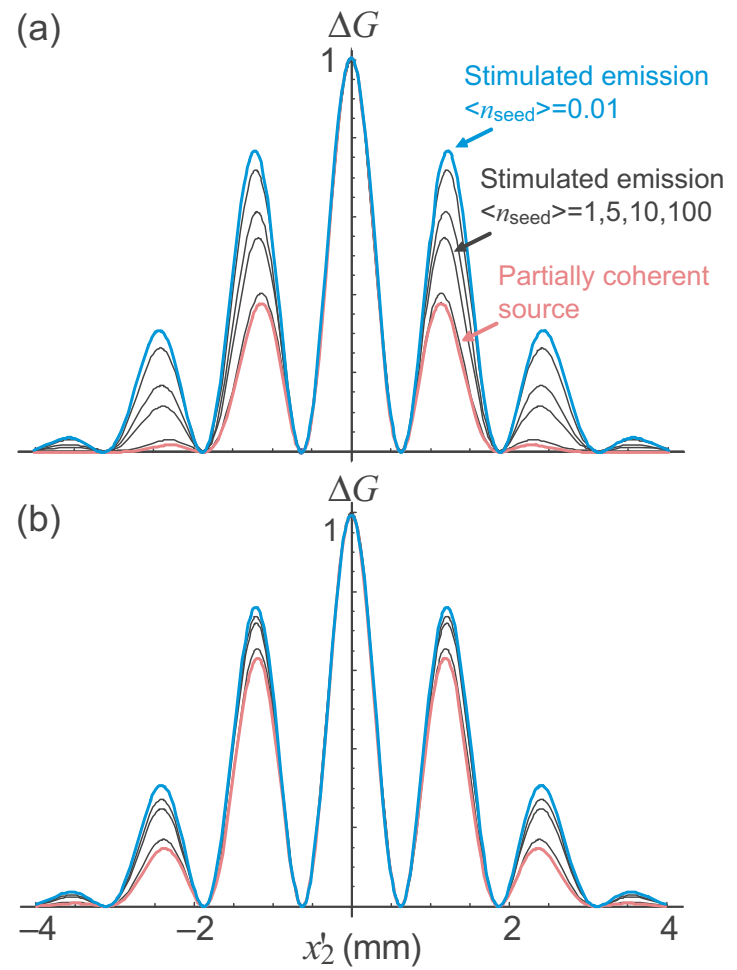

Fig. 2. (Color online) Effect of $l_{\text {seed }}$ on the reconstruction of a diffraction pattern of a double slit of width $b=0.08 \mathrm{~mm}$ and distance between the slits $d=0.2 \mathrm{~mm}$, showing the normalized coherence parameter $\Delta G_{\mathrm{PDC}+\text { seed }}\left(x_{1}^{\prime}=0, x_{2}^{\prime}\right)$ as scanned in the detector plane $x_{2}^{\prime}$. The results for a stimulated emission source are shown for various intensities of the seed (lower intensities of the seed correspond to the curves closer to the PDC result). The diffraction pattern/visibility obtained using only an equivalent partially coherent source is also shown. The optical setup has a lens of focal length $f=500 \mathrm{~mm}$ and wavelength $\lambda=702 \mathrm{~nm}$. $L=1 \mathrm{~mm}, \sigma_{p}=0.1$. (a) $l_{\text {seed }}=40 \mu \mathrm{m}$; (b) $l_{\text {seed }}=20 \mu \mathrm{m}$. 
ing stimulated PDC are closer to the diffraction pattern of the partially coherent source. We observed a dependence of the stimulated emission diffraction pattern on the coherence length of the seed while other parameters remained fixed.

In Fig. 2(b) where the coherence length is shorter than in Fig. 2(a), the difference between the diffraction pattern for PDC and stimulated PDC is much less significant. To understand this behavior we studied $\Delta G_{\mathrm{PDC}+\text { seed }}\left(x_{1}^{\prime}=0, x_{2}^{\prime}\right.$ $=1.5$ ), varying the coherence length of the seed for different values of $\left\langle n_{\text {seed }}\right\rangle$, as shown in Fig. 3 . It is clear that the dependence of $\Delta G_{\mathrm{PDC}+\text { seed }}\left(x_{1}^{\prime}=0, x_{2}^{\prime}=1.5\right)$ on $l_{\text {seed }}$ varies according to the number of photons per mode of the seed. For very low intensities of the seed there is no dependence on the coherence length, given that the PDC contribution dominates, while for higher number of photons per mode of the seed, the dependence on the coherence length is increased up to a point where it is almost equivalent to the partially coherent source (approximately when $\left\langle n_{\text {seed }}\right\rangle=1000$ ). This behavior is explained by Eq. (17), when the term in Eq. (18) is not negligible: the term represents the overlap between the PDC spatial coherence and the injected seed partial coherence. We have considered practical imaging situations where the object should be larger than the speckle size, and thus did not observe changes in the diffraction pattern for stimulated and spontaneous PDC, or for a classical source, with respect to variation of the parametric gain, the length of the crystal, or the transverse size of the seed.

\section{VISIBILITY}

To better understand the results reported in Fig. 3 and the advantage of using a stimulated PDC source, we calculated the visibility and compared it with the analogous setup using a partially coherent source. The definition of visibility is given in general by

$$
V\left(x_{1}^{\prime}, x_{2}^{\prime}\right)=\frac{\Delta G\left(x_{1}^{\prime}, x_{2}^{\prime}\right)}{\Delta G\left(x_{1}^{\prime}, x_{2}^{\prime}\right)+\left\langle n_{1}\left(x_{1}^{\prime}\right)\right\rangle\left\langle n_{2}\left(x_{2}^{\prime}\right)\right\rangle}
$$

where

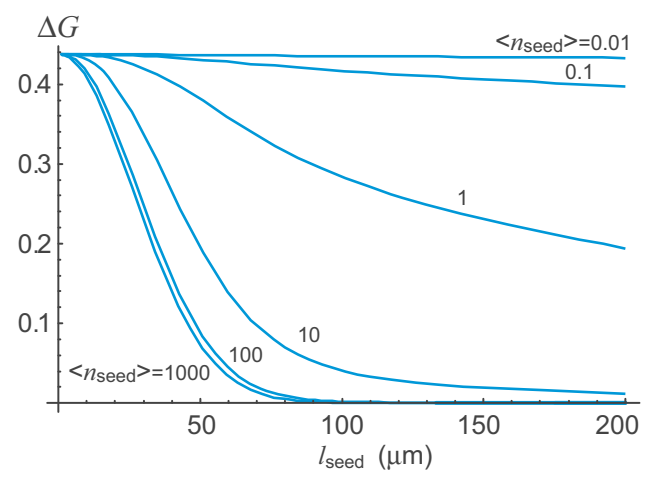

Fig. 3. (Color online) Normalized coherence parameter $\Delta G_{\mathrm{PDC}+\text { seed }}\left(x_{1}^{\prime}=0, x_{2}^{\prime}=1.5\right)$ of a double slit of width $b=0.08 \mathrm{~mm}$ and distance between the slits $d=0.2 \mathrm{~mm}$ versus the $l_{\text {seed }}$, for $\left\langle n_{\text {seed }}\right\rangle$ photons per mode as shown.

$$
\left\langle n_{j}\left(x_{j}^{\prime}\right)\right\rangle=\int \mathrm{d} x_{1} \mathrm{~d} x_{2} h_{j}\left(x_{1}, x_{j}^{\prime}\right) h_{j}^{*}\left(x_{2}, x_{j}^{\prime}\right) G^{(1)}\left(x_{1}, x_{2}\right), \quad j=1,2
$$

are the mean photon numbers measured at the detection planes. In the case of stimulated PDC, with a seed in arm 1 and following the same formalism,

$$
\begin{aligned}
\left\langle n_{1}\left(x_{1}^{\prime}\right)\right\rangle= & \frac{1}{2 \pi} \int \mathrm{d} q \mathrm{~d} x_{1} \mathrm{~d} x_{2} h_{1}\left(x_{1}, x_{1}^{\prime}\right) h_{1}^{*}\left(x_{2}, x_{1}^{\prime}\right) e^{-i q\left(x_{1}-x_{2}\right)} \\
& \times\left\{\left|V_{1}(-q)\right|^{2}\left[1+\widetilde{G}^{(1)}\left(x_{1}, x_{2}, q\right)\right]+\widetilde{G}^{(1)}\left(x_{1}, x_{2}, q\right)\right\},
\end{aligned}
$$

and

$$
\begin{aligned}
\left\langle n_{2}\left(x_{2}^{\prime}\right)\right\rangle= & \frac{1}{2 \pi} \int \mathrm{d} q \mathrm{~d} x_{1} \mathrm{~d} x_{2} h_{2}\left(x_{1}, x_{2}^{\prime}\right) h_{2}^{*}\left(x_{2}, x_{2}^{\prime}\right) e^{-i q\left(x_{1}-x_{2}\right)} \\
& \times\left|V_{2}(-q)\right|^{2}\left[1+\widetilde{G}^{(1)}\left(x_{1}, x_{2}, q\right)\right] .
\end{aligned}
$$

Figure 4 shows the visibility of the double-slit diffraction pattern obtained using stimulated PDC for different values of $\left\langle n_{\text {seed }}\right\rangle=0.1,1,10,100$, for PDC and partially coherent sources. The diffraction patterns of Fig. 4 corresponding to the conditions of Fig. 3 show that, for $l_{\text {seed }}$ $=20 \mu \mathrm{m}$, the visibility increases. In both cases the maximum visibility of the stimulated $\mathrm{PDC}$ is greater than 0.5 even for $\left\langle n_{\text {seed }}\right\rangle=0.1$. The visibility for the very low-gain regime is also shown $\left(\sigma_{p}=0.05\right)$; in this case, for stimulated PDC, the visibility is greater than 0.5 even for $\left\langle n_{\text {seed }}\right\rangle=1$. For higher gain $\left(\sigma_{p}=0.5\right)$ we see that the visibility drops, but even in this case, by reducing the coherence length of the seed and the number of photons per mode, the stimulated PDC visibility is higher than with the partially coherent source.

Figure 5 shows the maximum visibility for stimulated PDC versus the intensity of the seed, the coherence length of the seed, the length of the crystal, and the parametric gain. The visibility diminishes for high parametric gain as expected, and with longer crystals (thus longer coherence of the PDC). The important result for practical use of a stimulated PDC source in ghost imaging is an improvement of the visibility for low photon number per mode of the seed, and for low coherence length of the seed. In particular the dependence of the visibility on the coherence length of the seed, in the stimulated process, is opposite to that for the case where only a partially coherent source is used [13], where the visibility increases with the coherence length. This effect is dependent on the length of coherent overlap between the spatial coherence of the PDC process with the coherence of the injected seed. The result is particularly significant because it proves that stimulated PDC allows improvement of both visibility and resolution at the same time, while this is not possible in the case of a partially coherent source. Moreover, reducing the transverse size of the seed improves the visibility. This behavior is similar to results for classical sources. 

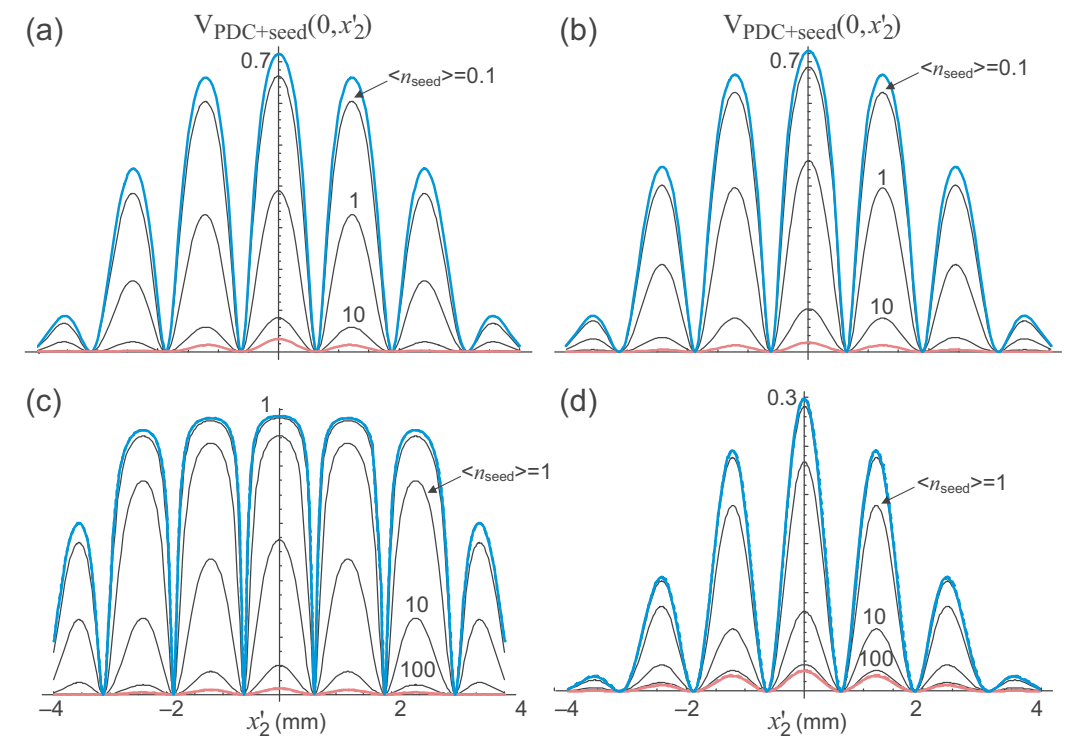

Fig. 4. (Color online) Visibility of the diffraction pattern of a double slit of width $b=0.08 \mathrm{~mm}$ and distance between the slits $d$ $=0.2 \mathrm{~mm}$, at position $x_{2}^{\prime}$ in the detector plane, for different conditions: (a) $\sigma_{p}=0.1, l_{\text {seed }}=40 \mu \mathrm{m}, L=1 \mathrm{~mm}$; (b) $\sigma_{p}=0.1, l_{\text {seed }}=20 \mu \mathrm{m}, L$ $=1 \mathrm{~mm}$; (c) $\sigma_{p}=0.05, l_{\text {seed }}=2 \mu \mathrm{m}, L=0.5 \mathrm{~mm}$; (d) $\sigma_{p}=0.5, l_{\text {seed }}=2 \mu \mathrm{m}, L=0.5 \mathrm{~mm}$. The results for a stimulated emission source are shown for various intensities of the seed, where lower intensities of the seed correspond to curves closer to the upper (PDC) curve in each case. The diffraction visibility obtained from using only an equivalent partially coherent source is also shown (lowest curve in each case). The optical setup has a lens of focal length $f=500 \mathrm{~mm}$ and $\lambda=702 \mathrm{~nm}$.
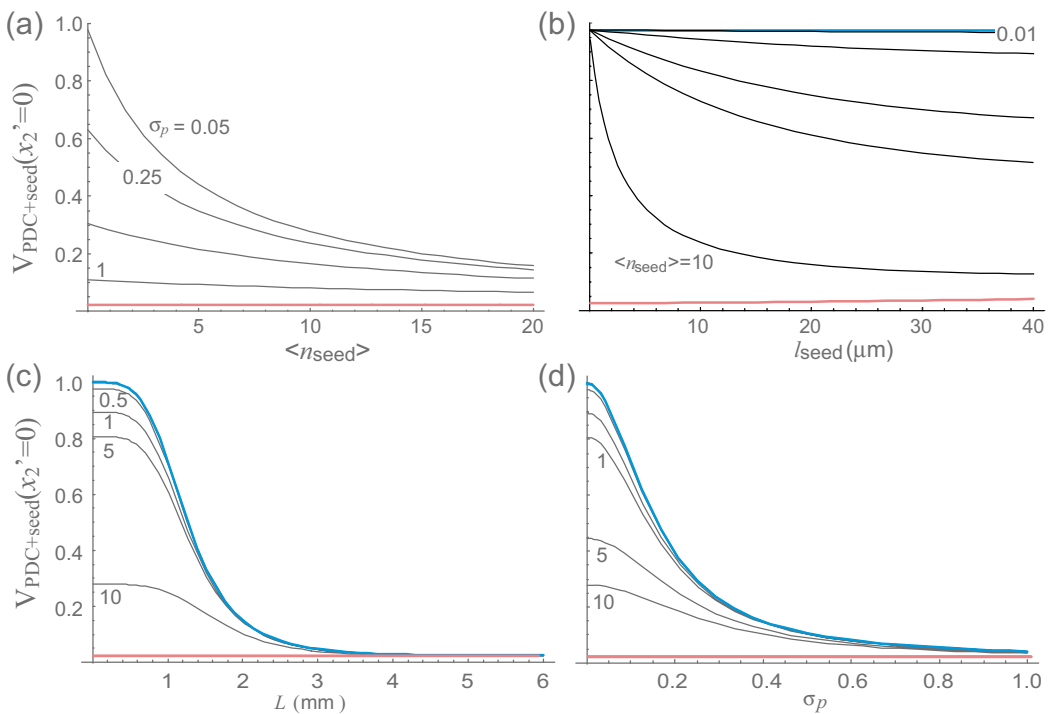

Fig. 5. (Color online) Maximum visibility of a double slit diffraction pattern for a stimulated PDC source versus (a) the mean number of photons of the seed for different values of the parametric gain $\sigma_{p}$, for $l_{\text {seed }}=6 \mu \mathrm{m}$; (b) the coherence length of the seed for different values of $\left\langle n_{\text {seed }}\right\rangle$ and for a fixed parametric gain $\sigma_{p}=0.1, L=0.5 \mathrm{~mm}$, and $l_{\text {seed }}=6 \mu \mathrm{m}$; (c) the crystal length for different values of $\left\langle n_{\text {seed }}\right\rangle$ $\left(l_{\text {seed }}=6 \mu \mathrm{m}\right)$; (d) the parametric gain for different values of $\left\langle n_{\text {seed }}\right\rangle\left(l_{\text {seed }}=6 \mu \mathrm{m}\right)$. The visibility with only PDC is shown as the upper curve in each case, and for an equivalent partially coherent source (curve at small $V$ ).

\section{CONCLUSION}

We have studied the properties of downconversion stimulated by a partially coherent seed, in terms of the visibility of a diffraction pattern reconstructed in a ghostimaging setup. In the approximation of a plane stationary pump, the visibility of ghost diffraction for stimulated emission can be adjusted by varying the intensity of the seed and its coherence length in both the low- and highgain regimes. In particular, for an injected pseudothermal source, it is possible to improve both the visibility and the resolution by reducing its transverse coherence. This property is characteristic of PDC sources and is retained for the stimulated PDC source outlined here. This source can be used to perform all two-photon imaging experiments, offering a wider range of parameters that can be adjusted for optimal visibility.

\section{REFERENCES}

1. R. Hanbury-Brown and R. Q. Twiss, "Correlation between photons in two coherent beams of light," Nature 177, 27-29 (1956).

2. A. V. Belinsky and D. N. Klyshko, "Two-photon optics- 
diffraction, holography and transformation of 2dimensional signals," Sov. Phys. JETP 78, 259-262 (1994).

3. T. B. Pittman, Y. H. Shih, D. V. Strekalov, and A. V. Sergienko, "Optical imaging by means of two-photon quantum entanglement,” Phys. Rev. A 52, R3429-R3432 (1995).

4. B. E. A. Saleh, A. F. Abouraddy, A. V. Sergienko, and M. C. Teich, "Duality between partial coherence and partial entanglement," Phys. Rev. A 62, 043816 (2000).

5. A. F. Abouraddy, B. E. A. Saleh, A. V. Sergienko, and M. C. Teich, "Role of entanglement in two photon imaging," Phys. Rev. Lett. 87, 123602 (2001).

6. R. S. Bennink, S. J. Bentley, and R. W. Boyd, "Two-photon coincidence imaging with a classical source," Phys. Rev. Lett. 89, 113601 (2002).

7. R. S. Bennink, S. J. Bentley, R. W. Boyd, and J. C. Howell, "Quantum and classical coincidence imaging," Phys. Rev. Lett. 92, 033601 (2004).

8. M. D'Angelo, Y-H. Kim, S. P. Kulik, and Y. Shih, "Identifying entanglement using quantum ghost interference and imaging," Phys. Rev. Lett. 92, 233601 (2004).

9. J. Cheng and S. Han, "Incoherent coincidence imaging and its applicability in X-ray diffraction," Phys. Rev. Lett. 92, 093903 (2004).

10. A. Gatti, E. Brambilla, M. Bache, and L. A. Lugiato, "Ghost imaging with thermal light: comparing entanglement and classical correlation," Phys. Rev. Lett. 93, 093602 (2004).

11. D. Magatti, F. Ferri, A. Gatti, M. Bache, E. Brambilla, and L. A. Lugiato, "Experimental evidence of high resolution ghost imaging and ghost diffraction with classical thermal light," Phys. Rev. Lett. 94, 183602 (2005).

12. G. Scarcelli, V. Berardi, and Y. Shih, "Can two-photon correlation of chaotic light be considered as correlation of intensity fluctuations?," Phys. Rev. Lett. 96, 063602 (2006).

13. Y. J. Cai and S. Y. Zhu, "Ghost interference with partially coherent radiation," Opt. Lett. 29, 2716-2718 (2004).

14. J. Xiong, De-Zhong Cao, F. Huang, H.-G. Li, X.-J. Sun, and $\mathrm{K}$. Wang, "Experimental observation of classical subwavelength interference with a pseudothermal light source," Phys. Rev. Lett. 94, 173601 (2005).

15. D. Zhang, Y-H. Zhai, L-A. Wu, and X-H. Chen, "Correlated two-photon imaging with true thermal light," Opt. Lett. 30, 2354-2356 (2005).
16. M. D'Angelo, A. Valencia, M. H. Rubin, and Y.. Shih, "Resolution of quantum and classical ghost imaging," Phys. Rev. A 72, 013810 (2005).

17. F. Ferri, D. Magatti, A. Gatti, M. Bache, E. Brambilla, and L. A. Lugiato, "High-resolution ghost image and ghost diffraction experiments with thermal light," Phys. Rev. Lett. 94, 183602 (2005).

18. M. Bache, E. Brambilla, A. Gatti, and L. A. Lugiato, "Ghost imaging using homodyne detection," Phys. Rev. A 70, 023823 (2004).

19. M. Bache, D. Magatti, F. Ferri, A. Gatti, E. Brambilla, and L. A. Lugiato, "Coherent imaging of pure phase object with classical incoherent light," Phys. Rev. A 73, 053802 (2006).

20. S. Crosby, S. Castelletto, C. Aruldoss, R. E. Scholten, and A. Roberts, "Modelling of classical ghost images obtained using scattered light," New J. Phys. 9, 285-295 (2007).

21. Y. Bai, H. Liu, and S. Han, "Transmission area and correlated imaging," Opt. Express 10, 6062-6068 (2007).

22. A. Kolkiran and G. S. Agarwal, "Quantum interference using coherent beam stimulated parametric downconversion," Opt. Express 10, 6479-6485 (2008).

23. E. Puddu, A. Andreoni, I. P. Degiovanni, M. Bondani, and S. Castelletto, "Ghost imaging with intense fields from chaotically seeded parametric downconversion," Opt. Lett. 32, 1132-1134 (2007).

24. I. P. Degiovanni, M. Bondani, A. Andreoni, E. Puddu, and M. Paris, "Intensity correlations, entanglement properties, and ghost imaging in multimode thermal-seeded parametric down-conversion: Theory,” Phys. Rev. A 76, 062309 (2007).

25. S. Thanvanthri and M. H. Rubin, "Ghost interference with an optical parametric amplifier," Phys. Rev. A 70, 063811 (2004).

26. G. S. Agarwal, R. W. Boyd, E. M. Nagasako, and S. J. Bentley, "Nonclassical two-photon interferometry and lithography with high-gain parametric amplifiers," Phys. Rev. A 64, 043802 (2001).

27. E. Brambilla, M. Bache, A. Gatti, and L. A. Lugiato, "Simultaneous near-field and far-field spatial quantum correlations in the high-gain regime of parametric downconversion," Phys. Rev. A 69, 023802 (2004). 